\title{
Mercè Rodoreda à Genève
}

\section{Mariàngela Vilallonga}

\section{Résumé}

Ce travail suit la géographie littéraire de l'écrivaine catalane Mercè Rodoreda à travers la ville de Genève pendant les années qu'elle y vécut, depuis 1954 jusqu'à 1979, par intermittence les sept dernières années. A partir de son oeuvre, spécialement de la narration "Paralysie", on peut fixer sur la carte de Genève la vie et l'oeuvre de Rodoreda, les endroits vécus et les endroits décrits dans sa production littéraire. Enfin, c'est un itineraire littéraire de Rodoreda à Genève.

\begin{abstract}
This work follows the literary geography of the Catalan writer Mercè Rodoreda through the city of Geneva during the years she lived there from 1954 to 1979 to the intermittent last seven years. From his work, especially the narration "Paralysis", can be fixed on the map of Geneva life and work of Rodoreda, the experienced places and places described in his literary production. Finally it is a Rodoreda literary route to Geneva.
\end{abstract}

\section{Citer ce document / Cite this document :}

Vilallonga Mariàngela. Mercè Rodoreda à Genève. In: Le Globe. Revue genevoise de géographie, tome 156, 2016. Italie. Paysage et identité. pp. 157-170;

doi : 10.3406/globe.2016.7402

http://www.persee.fr/doc/globe_0398-3412_2016_num_156_1_7402

Document généré le 19/04/2017 


\section{MERCE RODOREDA A GENEVE}

\section{Mariàngela VILALLONGA}

Université de Gérone

Résumé : Ce travail suit la géographie littéraire de l'écrivaine catalane Mercè Rodoreda à travers la ville de Genève pendant les années qu'elle y vécut, depuis 1954 jusqu'à 1979, par intermittence les sept dernières années. A partir de son oeuvre, spécialement de la narration "Paralysie", on peut fixer sur la carte de Genève la vie et l'œuvre de Rodoreda, les endroits vécus et les endroits décrits dans sa production littéraire. Enfin, c'est un itineraire littéraire de Rodoreda à Genève.

Mots-clés : Patrimoine littéraire, géographie littéraire, itinéraire littéraire, Mercè Rodoreda, Genève.

Abstract : This work follows the literary geography of the Catalan writer Mercè Rodoreda through the city of Geneva during the years she lived there from 1954 to 1979 to the intermittent last seven years. From his work, especially the narration "Paralysis", can be fixed on the map of Geneva life and work of Rodoreda, the experienced places and places described in his literary production. Finally it is a Rodoreda literary route to Geneva.

Keywords : Literary heritage, literary geography, literary route, Mercè Rodoreda, Geneva.

Née le 10 octobre 1908 à Barcelone et décédée le 13 avril 1983 à Gérone, l'écrivaine catalane Mercè Rodoreda se vit contrainte à l'exil après la guerre civile espagnole, d'abord à Paris, puis à Genève.

Si le couple formé par Armand Obiols - pseudonyme de Joan Prat - et Mercè Rodoreda est parti vivre en Suisse, c'est parce qu'Armand avait trouvé un emploi bien payé comme traducteur à l'ONU. C'est donc apparemment en 1954 qu'ils s'installèrent au 19, rue du Vidollet, tout près du siège de cet organisme international. Dans une lettre de Genève datée du 29 octobre 1956, l'écrivaine se confie à son amie Anna Murià, qui avait épousé le poète Agustí Bartra : "Nous sommes à Genève depuis deux ans et demi, quand nous n'étions venus que pour un contrat de trois semaines pour Obiols. [...] Même sans tenir compte de toutes les choses désagréables que j'ai vécues à Genève, je me suis toujours sentie exilée, ici." Dans une interview désormais célèbre qu'elle accorde à Baltasar 
Porcel en 1972, Mercè Rodoreda décrit en ces mots son exil obligé au bord du lac Léman :

Je vis dans un très joli studio qui donne sur un parc ; il y a un immeuble de sept étages de l'autre côté, mais il est assez loin. D'un côté, une partie du lac, et de l'autre, le Salève. Vue de ma terrasse, c'est une montagne assez laide. Elle est pelée à beaucoup d'endroits et ça lui donne l'air malade. Les jours dégagés, je vois la pointe du mont Blanc.

\section{Des souvenirs et une nouvelle}

Sous l'épigraphe "Souvenirs de Paris et de Genève", l'inventaire des archives de la Fondation Mercè Rodoreda de l'Institut d'Estudis Catalans fait état d'un document d'une seule page où l'écrivaine énumère les endroits qu'elle fréquentait pendant son long séjour dans la ville suisse : les cinémas Hollywood, Nord-Sud (rue de la Servette) et Central ; le café Le Radar et la salle de concert du Victoria Hall, entre autres. Elle ajoute, en espagnol, une brève description décousue de Genève. A elles seules, ces notes (Rodoreda, 2008:108) suffiraient à retracer tout un itinéraire "rodorédien".

Mais il y a aussi une nouvelle, "Paralysie" - insérée dans le recueil Comme de la soie, publié pour la première fois à Barcelone en 1978 - où Genève sert de toile de fond au monologue intérieur de l'héroïne. A travers le filtre de la littérature, presque tous les éléments de la liste de Rodoreda, ainsi que quelques autres nouveaux, surgissent au cours de la narration. "Paralysie" débute par les deux derniers vers (en français) du dernier quatrain (le 109) des Contrerimes du poète français Paul-Jean Toulet (Pau, 1867 - Guéthary, 1920). Deux vers qui font allusion à la mort d'un autre poète, Nicolas-Joseph-Laurent Gilbert (Fontenay-le-Château, 1751 Paris, 1780) qui, selon la légende, aurait avalé une clé dans une crise de délire : "Il faut savoir mourir, Faustine, et puis se taire, / mourir comme Gilbert en avalant sa clé" (Toulet, 1979:154).

Sous la protection de Toulet, Rodoreda construit une histoire qui se déroule au moins entre deux temps et deux espaces : Barcelone et Genève. Les deux villes et les deux moments s'entrelacent tout au long d'une narration syncopée, vagabonde et pleine de ces interminables connexions

LE GLOBE - TOME 156 - 2016 
dont Rodoreda a le secret. Si tous les romans écrits à Genève se passent à Barcelone, cette nouvelle est, elle, le pont qui relie les deux villes et qui unit aussi la vie et l'œuvre de l'auteure. C'est ce récit qui va guider notre promenade littéraire dans la Genève de Rodoreda (fig. 1).

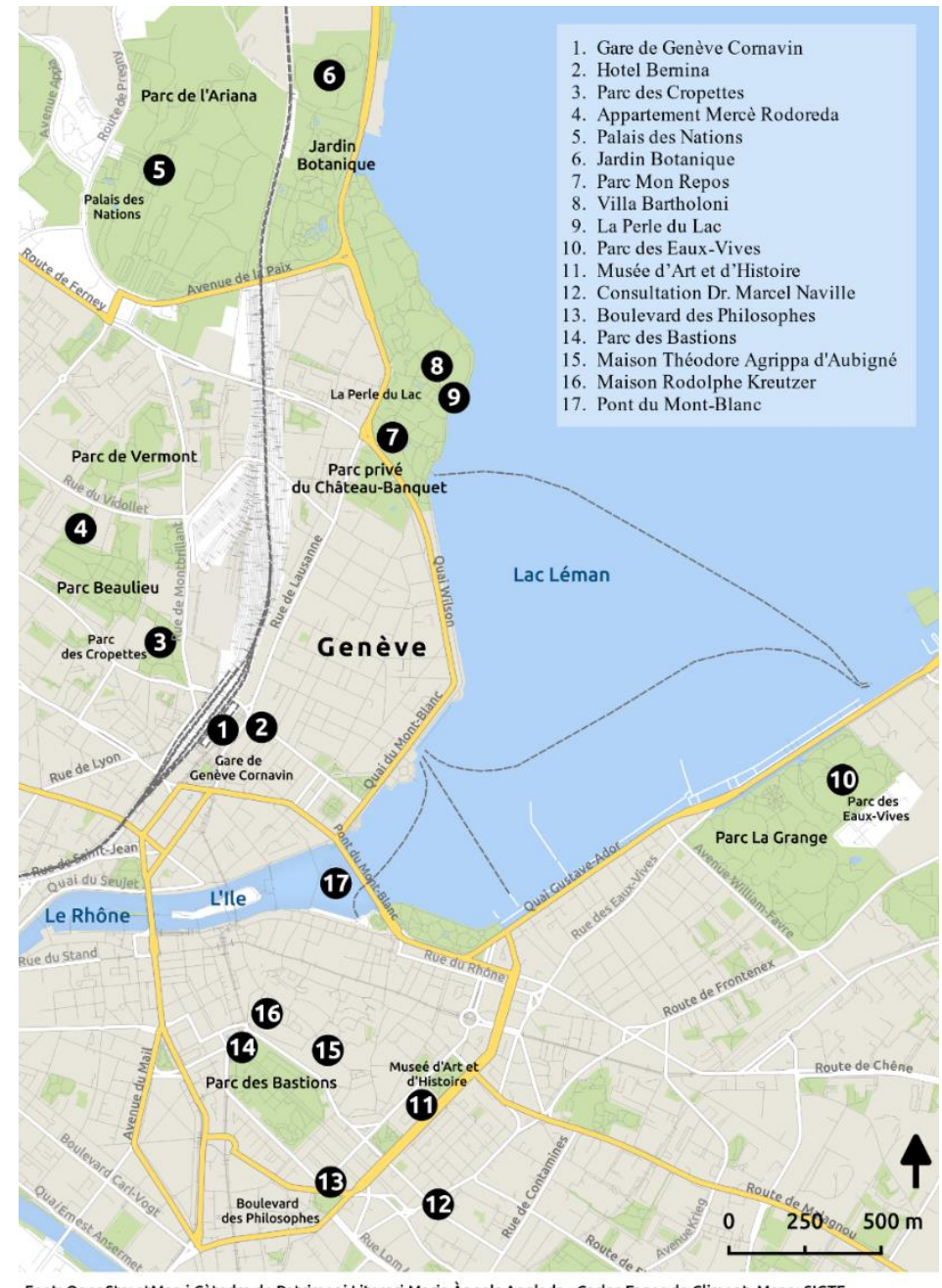

Fig. 1 : Carte littéraire de la Genève de Mercè Rodoreda 
Dans "Paralysie" (Rodoreda, 1991:125), il y a d'abord une fleur et puis, aussi, le souci de la langue. La narration commence par cette phrase : "J'ai regardé 'digitale' dans le dictionnaire." A la fin du récit, nous saurons qu'il y avait des digitales derrière les buis que l'on voyait de la terrasse de l'appartement de Genève. Rodoreda parle donc de cette ville, même si elle ne la nomme pas explicitement, lorsque l'héroïne, qui souffre d'une douleur au pied, se prépare pour aller consulter un médecin. Ensuite, ce sera l'évocation de Barcelone et d'un autre cabinet médical. Il y aura aussi Limoges et le souvenir d'un bras paralysé et d'une opération. Mais, quelques lignes après le début de l'histoire, c'est bien Genève qui apparaît, maintenant en toutes lettres :

Genève. Digitale. A feuilles linéaires. Vous êtes Genevois? Le gazon des parcs est presque brûlé et les arbres sont déjà dorés bien que nous soyons toujours en été. Une ville de feuilles, de chemins verts, de jardins gorgés de fleurs qui pousseraient toutes seules. Comme la voie du naturel, si difficile. Et de la spontanéité (Rodoreda, 1991:126).

Le médecin est genevois mais elle, l'héroïne de "Paralysie", se déclare catalane : "Méditerranéenne. Sirènes, dauphins et Ulysses à la pelle." Les deux cabinets, celui du médecin de Barcelone d'il y a longtemps et celui, bien plus récent, du médecin de Genève, se mélangent dans les pensées troublées et erratiques de cette femme. Les "autos" de la Barcelone d'avant-guerre deviennent tout à coup des "voitures", celles de la Genève des années soixante-dix. La femme de "Paralysie" demande un taxi de chez elle parce que l'appartement du 19, rue du Vidollet est tout près de la grande gare de Cornavin, dans le centre de Genève : "J'appelle un taxi. Cornavin. Il vient de la station de Cornavin. Je descends l'attendre. Le chauffeur ferme la portière, je cherche sur mon carnet d'adresses le numéro de la maison où je vais parce que je ne m'en souviens jamais. Et la nausée me prend. Peu sensible. Légère. Semblable à celle que me donnait l'odeur de vernis des tramways quand j'étais petite" (Rodoreda, 1991:127-8).

Le numéro de la maison où elle se dirige, celui dont elle n'arrive jamais à se souvenir, c'est celui du cabinet du docteur Marcel Naville (1910-1996), 15, rue de l'Athénée, à côté du boulevard des Philosophes, dans la Genève bourgeoise construite ou refaite au XIX ${ }^{\mathrm{e}}$ s., juste de l'autre côté de la ville, de l'autre côté du lac. Le fil du récit renvoie

LE GLOBE - TOME 156 - 2016 
l'héroïne à ses souvenirs barcelonais, ceux de son enfance, du temps où il y avait des tramways comme ceux qui circulent encore dans les rues ou sur les ponts de Genève par où passe le taxi :

Pont du Mont-Blanc. Le Salève est laid, dénudé par endroits. Mais au sommet, le paysage de crêtes enneigées est lunaire. La majesté des cimes, la solitude des glaciers, le ciel traversé d'aigles, d'ailes noires, de tempêtes de neige et d'ouragans. Une montagne changeante ; parfois lointaine, parfois à portée de main, nimbée de brouillard. Le brouillard de l'Arve au ras du fleuve, à ras de terre. Le pont des désespérés où se rejoignent l'Arve et le Rhône, où se mêlent leurs eaux claires et boueuses. Ceux qui se jettent du haut du pont sont déjà morts en touchant l'eau... L'idée du suicide m'incline à me croire importante, je me redresse sur mon siège et regarde passer Genève. [...] Une embardée du taxi me précipite contre la portière. Comment vais-je expliquer mon angoisse ? Et cette envie de hurler. Ce qu'il m'impose n'est pas bien. Boulevard des Philosophes*1 (Rodoreda, 1991:128-9).

\section{Bacon, Tolstoï, D'Aubigné et les seigneurs de la Réforme}

La Genève rive gauche et la Genève rive droite sont largement connectées au cœur de la ville par le pont du Mont-Blanc bordé de drapeaux. Le Salève, la montagne qui la poursuit où qu'elle aille, est toujours présent d'un bout à l'autre de Genève. Toujours là, laid, toujours là. D'un côté, le Jet d'Eau, de cent-trente mètres de haut ; de l'autre, l'île Rousseau. D'autres ponts et le suicide, savoir mourir. Elle a demandé bien peu et elle a beaucoup donné, se plaint-elle. Mais le temps passe et tout devient moins important. Elle est au milieu de sa vie : "J'ai fermé la baie vitrée. Je suis demeurée un moment derrière les carreaux à observer le Salève en relief" (Rodoreda, 1991:133). Encore des fleurs, le jasmin royal du roi Jaume, encore des digitales, encore des jardins et une citation de Francis Bacon (1561-1626) tirée de The Essays, au début du chapitre "Of gardens" : "God, first, planted a garden." Josep Carner nous a laissé la traduction en catalan de ces Essais de Bacon, publiée en 1972. Des fleurs, des fleurs et encore des fleurs, des fleurs pour de vrai, comme celles sur lesquelles elle écrit à Genève et qui apparaîtront plus tard, dans le recueil Voyages et Fleurs, avec les voyages qu'elle écrira à Romanyà. A Genève, les fleurs sont partout, de toutes les couleurs, de toutes les variétés, et on dirait des vraies, pas comme les fausses "Fleurs pour de vrai" du recueil.

LE GLOBE - TOME 156 - 2016 
Lorsque, des années plus tard, Rodoreda s'achètera un terrain à Romanyà de la Selva, elle y plantera des fleurs avant d'y construire sa maison. Mais son premier jardin fut un jardin de fleurs pour de vrai, à Genève.

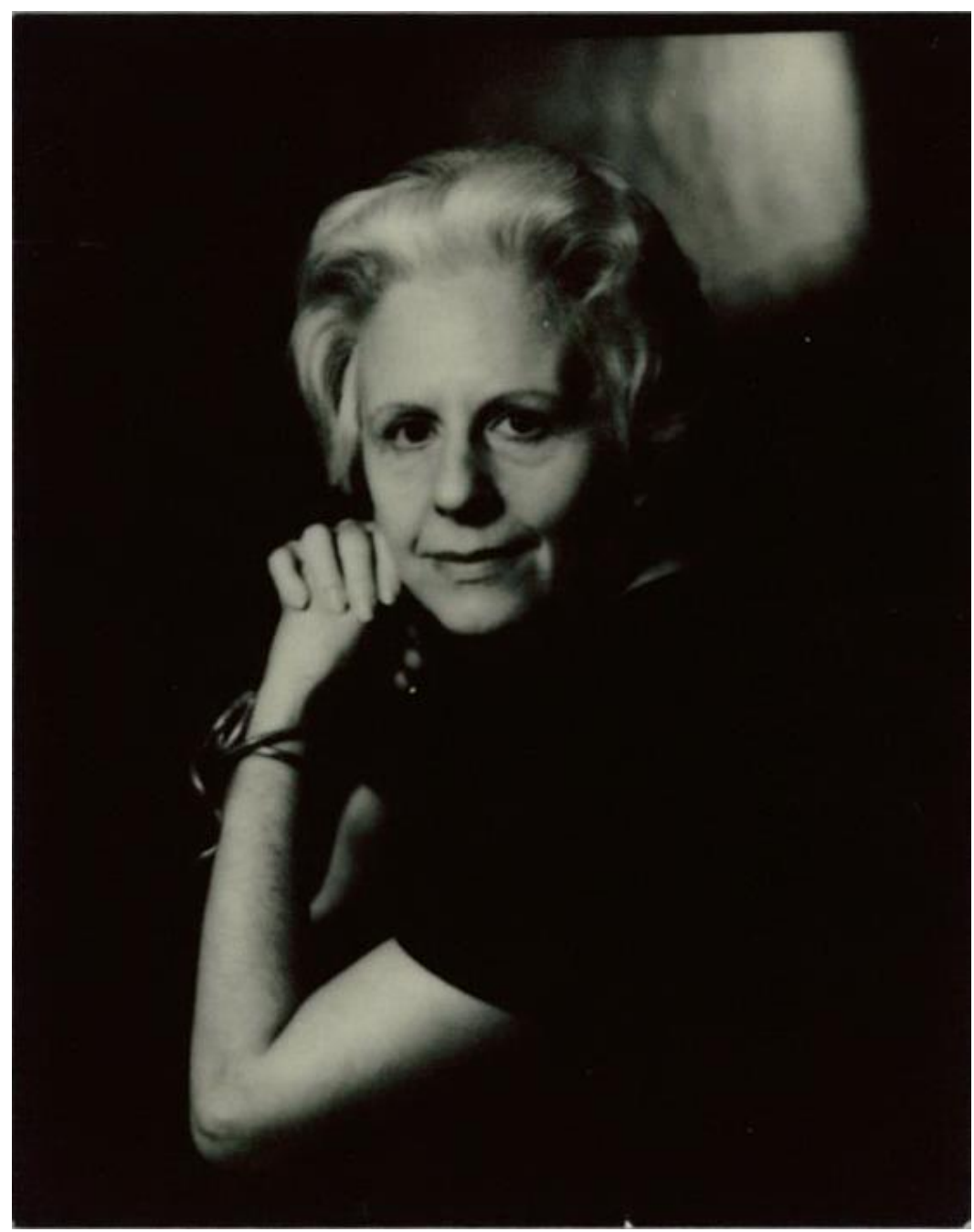

Fig. 2 : Mercè Rodoreda. Photographie Studio Mandanis, Genève, 11 février 1970. Arxiu de la Fundació Mercè Rodoreda de l'Institut d'Estudis Catalans

LE GLOBE - TOME 156 - 2016 
Voyages et fleurs, Romanyà et Genève. "Ecrire, c'est être seul." Et les digitales sont vénéneuses.

Puisque les fleurs c'est moi, je m'y fonds et je peux m'en passer. Je viens de mettre la Sonate à Kreutzer. Je ne suis pas dans la salle d'attente du médecin, je ne pense à rien de ce que je dis, je ne suis pas émue. J'écris. J'écris et ne parviens pas à communiquer l'intense mélange de sensations que je voudrais livrer. Personne n'atteint la vraie vie. Tentatives, épreuves. Essais. Ruses de Sioux, l'Indien le plus astucieux. Néant. La sonate sur le tourne-disque et les feuilles à remplir ! Je parle de moi. Et je n'en parle pas du tout. Quand quelqu'un de très intelligent dira : Voilà enfin dévoilées toutes ses astuces d'écrivain qui veut mais qui n'arrive pas... et comme elle avoue, Seigneur... elle se retrouvera les mains vides. Je ne donnerai rien. Je parlerai de moi sans cesse et ne donnerai rien. Je suis paralysie. Pourtant mes mains resteront vides car personne ne dit la vérité ; d'ailleurs, elle est insaisissable. Glissante comme une anguille. Et la Sonate à Kreutzer me rappelle ma première lecture de Tolstoï. Cette exaltation. Je cesse d'écrire et j'écoute. C'est vrai qu'il fait chaud, que j'écoute une sonate, que les jardinières des balcons sont remplies de pétunias et de géraniums rouges. A Genève. Et il est vrai que je serais moi si je n'étais pas une autre (Rodoreda, 1991:136-137).

Nexus rerum. Une nouvelle connexion avec la ville réelle. A partir de 1826 et jusqu'à sa mort en 1831, Rodolphe Kreutzer vécut au 16, promenade de Saint-Antoine, tout près de la maison du médecin. En 1803, sept ans après leur rencontre, Ludwig van Beethoven dédiera au célèbre violoniste la sonate pour violon et piano qui porte son nom. Il semblerait que Kreutzer n'interpréta jamais cette sonate pleine de fougue. Des années plus tard, terriblement ému par la sonate de Beethoven qu'il qualifiera d'"incitation à la destruction de la morale", Léon Tolstoï condamnera le mariage dans une nouvelle du même titre, La Sonate à Kreutzer, que Rodoreda avait, en français, dans sa bibliothèque.

Rodoreda aurait dit de Genève que "c'est une ville très ennuyeuse, idéale pour écrire." La solitude de Genève, pouvoir écrire à Genève, pouvoir peindre, pouvoir observer, avoir du temps pour soi à Genève. C'est qu'Obiols était parti vivre à Vienne car il devait y aller trop souvent. A cause du travail, lui disait-il. Alors elle, toute seule, elle écrivait comme jamais. Elle le refera plus tard, au milieu des fleurs, à Romanyà, où elle

LE GLOBE - TOME 156 - 2016 
achèvera certaines œuvres commencées à Genève, au bord du lac Léman. D'ailleurs, que peut-elle faire d'autre, si elle est seule ? Elle a du temps pour elle. Comme à Romanyà.

Tout s'éloigne. J'ignore ce que je dis au médecin qui me regarde et je lui demande s'il veut savoir autre chose. Il ne répond pas. Ça pourrait donner une scène maniérée. Parler du parfum des roses dans le vase, de la qualité des pétales, une rose d'automne est plus qu'une autre exquise*, mais à quatre pas de là le violon se déchaîne parce qu'un homme à part traça autrefois maints gribouillis sur cinq lignes, $\mathrm{j}$ 'aurais pu dire sur une portée mais ce ne serait pas mon genre : cinq lignes (Rodoreda, 1991:138).

Encore des fleurs. Maintenant, ce sont des roses. Et aussi une citation sur la rose. Elle n'est pas de Ronsard mais de Théodore Agrippa d'Aubigné, qui vécut les dernières années de sa vie à Genève pour y mourir finalement en 1630, exilé après avoir participé activement aux guerres de religion. Une plaque nous indique la maison qu'il occupa rue de l'Hôtel-de-Ville, pas loin du cabinet du Dr Naville, si l'on emprunte le chemin qui traverse la Vieille Ville.

Il m'en a coûté d'aimer Genève. Je m'ennuyais à mourir sans le Louvre, les musées, les vieilles rues, les grandes avenues. Rue de Prony*. Enracinée à Paris, je ne sais combien d'années passées à Paris. Le jardin du Luxembourg et tant de saintes reines de France... il manque un doigt à sainte Clotilde, j'ignore si c'est à la main droite ou à la main gauche. Et une voix me parvient, sortant d'une bouche perchée en haut d'une blouse blanche qui s'encadre dans la porte-fenêtre; quand j'affirme que le Salève est laid, tout l'orgueil national se révolte. Mais d'en haut. Ce qu'on voit d'en haut. L'enchantement s'évanouit. Cette sonate me perturbe au lieu de m'inspirer. Admirable, trop, beaucoup trop. Stop ! Que cette brute de violon s'arrête. Non, ce n'est pas le jour des roses rouges que j'ai assuré au médecin que le Salève était la montagne la plus laide du monde. C'est la faute de la sonate si j'ai écrit ça. Je serre les dents : le ruban de la machine s'est coincé, je le décoince. Je suis... Nous cessons tous de vivre à douze ans. Voilà pourquoi j'ai toujours la passion des fleurs (Rodoreda, 1991:138-9).

Beethoven, Tolstoï : la Sonate à Kreutzer est la musique de fond de la Genève "rodorédienne". Stendhal, de passage à Genève en 1830, nous

LE GLOBE - TOME 156 - 2016 
avait déjà dit que le Salève était laid. Quand le romancier français vit les dégâts que l'exploitation excessive des carrières avait infligés à la montagne, il trouva que ce vulgaire rocher dénudé aurait mieux fait de disparaître. Plus ou moins comme celui que décrit Rodoreda.

Voilà désormais que j'aime Genève et le Salève dénudé ; qu'après y avoir étouffé des années durant, je l'ai enfin découverte. Reprenons, c'est maintenant que je signale au médecin la laideur du Salève. Nous passons dans le cabinet de consultation. Une vitrine et des instruments chirurgicaux, des boules de coton dans un pot en verre, une bascule, un divan... Je me déshabille. C'est la combinaison noire ! J'entends encore ma voix expliquer comment a débuté mon mal au pied : au parc des Cropettes, dans une montée. Un élancement à la cheville comme si un morceau de verre s'était brisé dedans et les éclats s'étaient enfoncés dans les cartilages. Impossible. Ce serait un rhumatisme et ce ne sont que mes nerfs. Pourtant je lui cache que, lors de la montée dans le parc, tout mon être, toute ma peau, tissu dermique ou sous-cutané, tremblaient. Les nerfs se sont alors concentrés dans le pied gauche et, en arrivant chez moi, je boitais déjà. Qu'est-ce que tu as ? Je ne sais pas, j'ai dû me tordre le pied. J'ai mal. Sans m'en apercevoir (Rodoreda, 1991:141).

Le chemin qui va du parc des Cropettes à la rue du Vidollet n'est pas long, même pour une cheville tordue. Dans le parc, un étang en forme de demi-lune. Une façon de nous rappeler, en miniature, le lac de Genève ? Il y a de grands arbres, des massifs de fleurs, des fleurs pour de vrai, comme dans tous les parcs de Genève. Nous nous trouvons de nouveau chez le Dr Naville, de l'autre côté de la ville, dans la vieille Genève.

Sur le mur d'en face, au bout du couloir, il y a un plan de Genève. Mais la vue, là-haut... L'infirmière m'ouvre la porte et je me retrouve dans la rue. La lumière est intense, le soleil proche du zénith, l'après-midi chaud. Je dois revenir d'ici trois semaines. J'ai peur de traverser la rue car dans ce pays les voitures roulent à toute allure, comme si personne n'allait arriver à temps. Si j'avais moins mal au pied, je descendrais en me promenant. Vous ne commettrez aucune folie ? Voilà ce qu'il a dit. Tous les autres troubles nerveux avaient été indolores. J'allais traverser les Bastions, voir la muraille couverte de lierre qui sert de décor aux maîtres de la Réforme. Genève : l'aigle, la clef. Je prends un taxi jusqu'à la maison. Je crierais" (Rodoreda, 1991:143). 
Le cabinet du Dr Naville est tout près du parc des Bastions, sous la promenade de la Treille, au pied de la vieille ville de la Genève fortifiée. Un mur couvert de lierre cache à moitié une plaque remémorant l'ancienne muraille, au parc des Bastions. Genève, ville de plaques aux murs, Genève, ville historiée. Le long mur des grands seigneurs de la Réforme : les grandes figures, Bèze, Calvin, Knox, Farel et, un peu à part, deux larges pilastres et les noms, rien que les noms, de Luther et de Zwingli, face à l'université ; au sol, des blasons en mosaïques avec l'ours, l'aigle et la clé, le lion, ceux de Berne, Genève et Edimbourg, lieux d'origine des quatre grands seigneurs. Le pavillon de musique, du $\mathrm{XIX}^{\mathrm{e}}$ s., au bout du parc, à côté de la place Neuve et du Grand Théâtre. Combien de fois s'y sera-telle arrêtée, au pavillon du parc, pour se reposer et peut-être pour prendre un thé avec des scones en contemplant les joueurs d'échecs géants ? Là, on a le choix, soit de descendre par le chemin le plus facile vers le pont du Mont-Blanc, soit de traverser la Vieille Ville en empruntant ses ruelles étroites et escarpées. Si l'on suit malgré tout le chemin de la Vieille Ville, on pourra contempler le Salève dans toute sa largeur depuis le banc insolitement long de la promenade de la Treille.

Retournons à notre nouvelle. Le jour de la visite chez le médecin, l'héroïne de "Paralysie" décide de rentrer rue du Vidollet en taxi :

En entrant chez moi, je respire. Je m'étends sur le lit et je pense à la clarté qui régnait dehors. J'ai mal au dos. L'éclairage du studio est différent car c'est l'entrée qui reçoit le soleil de plein fouet. J'allume une cigarette. Je vais devoir me rendre à la pharmacie pour acheter tout l'arsenal. Que t'a dit le médecin ? Rien. Mais qu'est-ce que tu as ? Je ne sais pas. Un rhumatisme. Je vais préparer le repas. Nous dînerons sur la terrasse. L'air est devenu dense et le Salève est embrumé. Sais-tu ce que sont des digitales ? Non. Tu vois ces taches de couleur par-delà les buis ? Ce sont des digitales. Comment le sais-tu ? Parce qu'elles ont la forme d'un doigtier. Lorsqu'il ne me regarde pas, je le regarde ; je sais que c'est risqué car la personne observée s'aperçoit toujours qu'elle est le point de mire, elle n'a pas besoin de regarder. Puis je détourne mon regard vers le lac où passe une voile blanche. Tu as mis un disque ? La Sonate à Kreutzer, tandis que j'écrivais. Je n'ai pas envie de musique. Je n'ai pas envie de petites notes rondes sur cinq lignes, pour le moment. La lumière devient grise et tous les réverbères s'allument en même temps (Rodoreda, 1991:144).

LE GLOBE - TOME 156 - 2016 


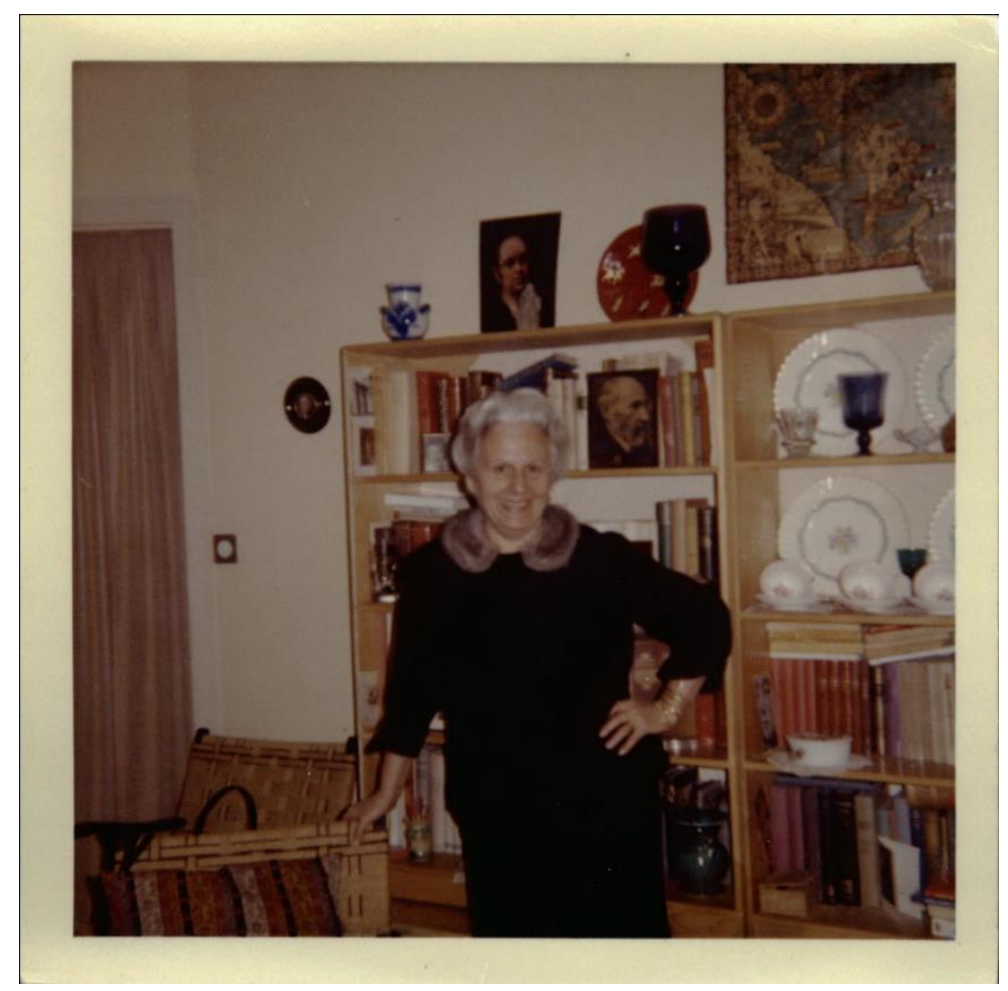

Fig. 3 : Mercè Rodoreda dans son appartement du 19, rue du Vidollet, 1967. Arxiu de la Fundació Mercè Rodoreda de l'Institut d'Estudis Catalans

Parmi la correspondance que conserve l'Institut d'Estudis Catalans, il y a six cartes postales du Dr Naville à Mercè Rodoreda, écrites entre 1961 et 1975. On y trouve aussi une lettre qu'elle lui envoie. "Personne ne dit la vérité ; d'ailleurs, elle est insaisissable." Dans une autre nouvelle inédite des archives de l'IEC, nous lisons à la fin :

Je suis allée prendre le thé à la terrasse du Palais des Nations sans passer par le bureau de M. Exceptionnellement, on voyait le mont Blanc, enneigé, le pic rose, un coucher de soleil aux nuages vénitiens, le lac bleu et rosé. Je fermais les yeux pour savourer tant de beauté. J'avais besoin d'amour et je ne savais où le trouver. Celui que j'avais s'était usé. Il restait de l'affection.

LE GLOBE - TOME 156 - 2016 
Dans ses notes en espagnol sur Genève, Rodoreda mentionne certains des invités qui dinèrent chez elle : Eugeni Xammar, Julio Cortázar et sa femme, le frère de Jorge Semprún. Elle termine sur une citation du prolifique écrivain suisse Gonzague de Reynold.

\section{La Perle du Lac}

Parmi le matériel légué par Mercè Rodoreda à l'Institut d'Estudis Catalans, se trouve une série de pages qui seraient le chapitre d'un roman à peine ébauché et qui se serait appelé La perla del llac. C'est aussi le nom d'un endroit de Genève où Rodoreda se rendait souvent et qui était situé au bord du lac, non loin de son appartement et des Nations Unies où elle allait déjeuner "tous les jours ; la vue de la salle à manger du dernier étage est merveilleuse". Après avoir passé l'entrée protégée par une grille en fer forgé où l'on peut lire le nom de l'endroit, on traverse un jardin exubérant pour arriver à un chalet typiquement suisse qui abrite un salon de thé et un restaurant. Sur la façade de la maison, ces grands mots de Jean-Jacques Rousseau : "Heureux celui qui sur ces bords peut longtemps se reposer. Heureux celui qui les revoit s'il a dû les quitter". Dans le prologue, devenu épilogue à l'édition française, de son roman Miroir brisé, Rodoreda fait une description détaillée de cet endroit qui, même s'il n'apparaît pas dans "Paralysie", est mentionné dans ses notes (Rodoreda, 2011:322-323) :

La Perle du Lac est un restaurant près du Léman. Fermé l'hiver, en été c'est un endroit enchanteur. Des dames et des messieurs, Genevois heureux d'être nés en Suisse, le paradis de l'Europe, prennent le thé sur la terrasse. Entre deux gorgées de thé, on voit l'eau sillonnée par des skieurs nautiques, par des bateaux à moteur, par des petites barques à voile, par des vapeurs blancs avec la cheminée noire et jaune qui font la traversée du lac. Le restaurant est entouré de jardins, de cèdres et de tilleuls centenaires, d'une débauche de fleurs, d'étendues de gazon sans le moindre brin qui ne soit d'un vert émeraude. Un soir, au coucher du soleil, une dame d'un certain âge descendit d'une Rolls, s'avança vers le petit mur au bord du lac et s'immobilisa à tel point qu'elle se confondait avec ce qui l'entourait. Elle portait des bijoux, chose rare chez une Genevoise : un large bracelet garni de brillants et de saphirs. Au bout d'un long moment, elle s'en alla. A quoi pouvait-elle penser tout en regardant les barques, le soleil et le ciel en miettes au-dessus de l'eau, le petit vapeur qui passait en faisant joyeusement résonner la sirène ? Pensait-elle à elle ? Revoyait-elle sa jeunesse ? Voyaitelle quelque chose ou bien était-elle si profondément perdue dans ses

LE GLOBE - TOME 156 - 2016 
souvenirs qu'elle ne voyait rien ? Plus tard, lorsque, sans faire quoi que ce soit pour y penser, j'ai pensé à elle, je ne savais pas si elle avait les cheveux blonds ou si elle les avait noirs, je l'ignore. Je me souvenais de ses yeux qui, un moment, rencontrèrent les miens ; des yeux d'une couleur indéfinie dans lesquels beaucoup de vie s'était peu à peu accumulée. Une image de raffinement un peu hors du monde, un peu différente de tout. En créant Teresa Goday de Valldaura, je lui ai donné les yeux de la dame du Léman.

La dame du muret près du lac imaginée par Rodoreda était peut-être madame Wilsdorf, l'épouse du fondateur de la célèbre manufacture suisse des montres Rolex. Celui-ci avait acquis la maison en 1923 à la famille Bartholoni, nom que porte encore aujourd'hui la villa voisine, de style palladien, qui abrite le musée d'Histoire des sciences. Lorsque madame Wilsdorf visita la propriété la première fois, elle s'exclama que c'était la perle du lac. En 1982, Rodoreda rédigea en ces termes un autre prologue, celui de la $26^{\mathrm{e}}$ édition de La Place du Diamant :

Il pourrait être intéressant d'expliquer la genèse de La Place du Diamant, mais peut-on expliquer la façon dont un roman prend forme, les pulsions qui en sont à l'origine, la volonté énorme qui réussit à nous faire continuer, à conclure avec acharnement ce que l'on a commencé si facilement ? Serait-il suffisant de dire que j'ai commencé à y penser à Genève en regardant le Salève ou en me promenant du côté de La Perle du Lac ? [...] Je l'ai écrit fébrilement, comme si chaque journée de travail était le tout dernier jour de ma vie. Je travaillais aveuglément ; je corrigeais l'aprèsmidi ce que j'avais écrit le matin, en essayant de ne pas perdre les rênes malgré l'urgence dans laquelle j'écrivais. [...] Ce fut une époque de grande tension nerveuse qui me rendit à moitié malade (Rodoreda, 1982:8).

Telle fut la vie fiévreuse d'écrivain que Rodoreda mena à Genève pendant de longues années. Dans une lettre à son fils datée du 28 septembre 1961 à Genève, elle déclare : "Je travaille beaucoup. Je me donne dix ans pour m'imposer et pour être un grand écrivain. J'y laisserai la vieille peau qui me reste car je me crève au travail." Fruits de tant de labeur, le recueil Vint-i-dos contes (1958), les romans La Place du Diamant (1962), Rue des Camélias (1966) et Jardí vora el mar (1967), un autre recueil de narrations, Une baleine nommée Cristina et autres nouvelles (1967), la réécriture d'Aloma (1968), les "Fleurs pour de vrai" de Voyages et Fleurs, une œuvre qui nous conduit à Romanyà de la Selva,

LE GLOBE - TOME 156 - 2016 
où Rodoreda écrira les "Voyages à quelques villages" et où elle achèvera aussi Miroir brisé, commencé à Genève en 1968.

Juste devant La Perle du Lac, il y a un embarcadère où l'on peut prendre une des Mouettes qui traversent le lac et qui nous laissera de l'autre côté, juste en face du parc des Eaux-Vives et de celui de La Grange et sa roseraie splendide que Rodoreda visitait souvent. Une autre bonne promenade dans la nature genevoise, avec tellement d'arbres et de fleurs qu'on dirait des vrais.

Le 29 janvier 1979, à Genève, Mercè Rodoreda écrit à Carme Manrubia, à Romanyà de la Selva: "Ma chère Carme : On viendra vider le studio le 5 ou le 6 et je prendrai le Talgo le 7 février. [...] Tu dois bien t'imaginer que je suis un peu énervée, mais je veux penser que tout ira bien. Je t'embrasse, Mercè." Au Senyal Vell, la maison de Romanyà de la Selva où Carme Manrubia reçut cette lettre, on conserve encore une facture de l'hôtel Bernina de Genève. Le 5, le 6 et le 7 février 1979, Mercè Rodoreda y occupa la chambre numéro 81. L'hôtel Bernina est situé en face de la gare de Cornavin, d'où sort le train de nuit qui mène directement à Gérone. Les déménageurs vidèrent le studio de la rue du Vidollet le 6 février 1979. C'en était fini des allées et venues entre Genève et Romanyà devenues de plus en plus fréquentes à partir de 1972.

\section{Bibliographie}

Rodoreda M., 1982, La Plaça del Diamant, Barcelona, Club Editor, 26 éd. (La Place du Diamant, Paris, Gallimard, 1971).

Rodoreda M., 1991, "Paralysie", in : Comme de la soie, Arles, Actes Sud, trad. par Christine Maintenant et Claude Bleton.

Rodoreda M., 2008, "Ginebra", Revista de Girona, T. 247, pp. 108-109.

Rodoreda M., 2011, Miroir brisé, Paris, Autrement, trad. par Bernard Lesfargues.

Toulet P.-J., 1979, Les Contrerimes, éd. de Michel Décaudin, Paris, Gallimard.

\section{Notes}

1. Les termes suivis d'un astérisque* sont en français dans le texte.

LE GLOBE - TOME 156 - 2016 